\title{
Modeling the Phase Behavior of the Membrane Binding Protein Annexin V
}

\author{
Massimo G. Noro, ${ }^{\dagger, \ddagger}$ Martin A. Bates, ${ }^{*, t, \S}$ Alain Brisson," and Daan Frenkel ${ }^{\dagger}$ \\ FOM Institute for Atomic and Molecular Physics, Kruislaan 407, \\ 1098 SJ Amsterdam, The Netherlands, Physical and Measurement Sciences Group, \\ Unilever Research Port Sunlight, Wirral $\mathrm{CH} 63$ 3J W, United Kingdom, Department of \\ Chemistry, University of Southampton, Southampton SO17 1BJ , United Kingdom, and \\ Department of Biophysical Chemistry, University of Groningen, Nijenborgh 4,
} 9747 AG Groningen, The Netherlands

Received October 18, 2001. In Final Form: J anuary 18, 2002

\begin{abstract}
The bulk thermodynamic properties of proteins originate from a varied and complex combination of interactions. We propose a simple model for the formation of ordered two-dimensional aggregates based on the interactions between pairs of annexin V molecules. Simulations of this model areshown toreproduce the experimental observations of a honeycomb (p6) and a triangular (p3) crystalline phase. The simulations indicate that the transition between these two phases is first order. While this model is extremely simple in that it relies only on hard body and short-range directional interactions, it nevertheless captures the essential physics of the interactions between the protein molecules and reproduces the phase behavior observed in electron microscopy and atomic force microscopy experiments.
\end{abstract}

\section{Introduction}

The phenomena of protein crystallization, aggregation, liquid-liquid phase separation, and self-assembly are of great importance in the field of protein structure determination ${ }^{1}$ and in the purification and stabilization of protein solutions. A detailed knowledge of the complex intra- and intermol ecular inter actions is required in order to fully understand the biol ogical properties of individual proteins or protein complexes. In contrast, greatly simplified models for the protein-protein interaction can be used to model the phase behavior and aggregation form of large numbers of proteins. Such a simplification is, in fact, essential when using computer simulations to predict the phase diagram of a protein solution. Here, we take such a point of view to construct a simpli fied model for the intermolecular interactions of annexin $\mathrm{V}$, a phospholipid binding protein, ${ }^{2}$ and usecomputer simulation techniques to determine the phase behavior of the model system.

The most reliable methods for crystallizing proteins in two dimensions rely either on the incorporation of membrane proteins within lipid bilayers ${ }^{3}$ or on thespecific anchoring of soluble proteins to lipid monolayers at the air-water interface ${ }^{4}$ or lipid bilayers at solid-liquid interfaces. ${ }^{5}$ The association of a protein to a planar

* Correspondingauthor. E-mail: bates@soton.ac.uk. Address for correspondence: Department of Chemistry, University of Southampton, Southampton SO17 1BJ , United Kingdom.

† FOM Institute for Atomic and Molecular Physics.

‡ Unilever Research Port Sunlight.

$\S$ University of Southampton.

"University of Groningen.

(1) McPherson, A. Preparation and Analysis of Protein Crystals; Krieger Press: Malabar, 1982.

(2) Demange, P.; Voges, D.; Benz,J .; Liemann, S.; Gottig, P.; Berendes, R.; Burger, A.; Huber, R. Annexin V: The key to understanding ion sel ectivity and vol tageregulation. Trends Biochem. Sci. 1994, 19, 272276.

(3) Ringler, P.; Heymann, B.; Engel A. Two-dimensional crystallisation of membrane proteins. In MembraneTransport; Baldwin, S. A., Ed.; Oxford University Press: New York, 2000; Chapter 10, pp 229268.

(4) Brisson, A.; Bergsma-Schutter, W.; Oling, F.; Lambert, O.; Reviakine, A. 2D crystallisation of proteins on lipid monolayers at the air - water interfaceand transfer toan el ectron microscopy grid. J . Cryst. Growth 1999, 196, 456-470. substrate seems to be sufficient for triggering selfassembly into two-dimensional (2D) crystals. This technology has been highly successful for annexin V, due to its property of binding specifically to negatively charged phospholipids such as phosphatidylserinein thepresence of $\mathrm{Ca}^{2+}$ ions. ${ }^{6,7}$ Annexin $\mathrm{V}$ molecules are monomeric in solution but, after binding to a membrane, spontaneously form tightly bound trimers. The process of trimerization is extremely rapid, and el ectron crystallography evi dence suggests that there is no significant change in the molecular conformation upon binding to the membrane. ${ }^{8}$

The membrane-bound trimers self-organize into two types of 2D crystals. ${ }^{9}$ Electron microscopy density maps probing the structure of these two phases are shown in Figure 1. These phases are based on the same building block, namely, the trimer, and show either p3 or p6 symmetry. Experiments on lipid monol ayers usingelectron microscopy (EM) and lipid bilayers using atomic force microscopy (AFM) indicate that p6 is the stable phase at low surface coverage. ${ }^{10,11}$ The $\mathrm{p} 6$ crystals are formed by

(5) Reviakine, I.; Bergsma-Schutter, W.; Brisson, A. Growth of protein 2D crystals on supported planar lipid bilayers imaged in situ by AFM . J . Struct. Biol. 1998, 121, 356-362.

(6) Uzgiris, E. E.; Kornberg, R. D. Two-dimensional crystallization technique for imaging macromolecules, with application to antigenantibody-complement complexes. Nature 1983, 301, 125-129.

(7) Brisson, A.; Olofsson, A.; Ringler, P.; Schmutz, M.; Stoylova, S. Two-dimensional crystallisation of proteins on planar lipid films and structure determination by electron crystallography. Bio. Cell. 1994, 80, 221-228.

(8) Oling, F.; Sopkova-De Oliveira Santos, J .; Govorukhina, N.; Mazeres-Dubut, C.; Bergsma-Schutter, W.; Oostergetel, G.; Keegstra, W.; Lambert, O.; L ewit-Bentley, A.; Brisson, A. Structure of membrane bound annexin A5 trimers: a hybrid cryo-em-X-ray crystallography study. J. Mol. Biol. 2000, 304, 561-573.

(9) Brisson, A.; Lambert, O.; Bergsma-Schutter, W. Two-dimensional crystallisation of soluble proteins on planar lipid films: a practical approach. In Crystallisation of Nucleic Acids and Proteins: A Practical Approach; Ducruix, A., Giegé, R., Eds.; Oxford University Press: New York, 1999; pp 341-363.

(10) Voges, D.; Berendes, R.; Burger, A.; Demange, P.; Baumeister, W.; Huber, R. Three-dimensional structure of membranebound annexin V. A correlative electron microscopy-X-ray crystallography study. J . Mol. Biol. 1994, 238, 199-213.

(11) Reviakine, I.; Bergsma-Schutter, W.; Mazeres-Dubut, C.; Govorukhina, N.; Brisson, A. Surfacetopography of the 3 and p6 annexin $\checkmark$ crystal forms determined by atomic force microscopy. J . Struct. Biol. 2000, 131, 234-239. 


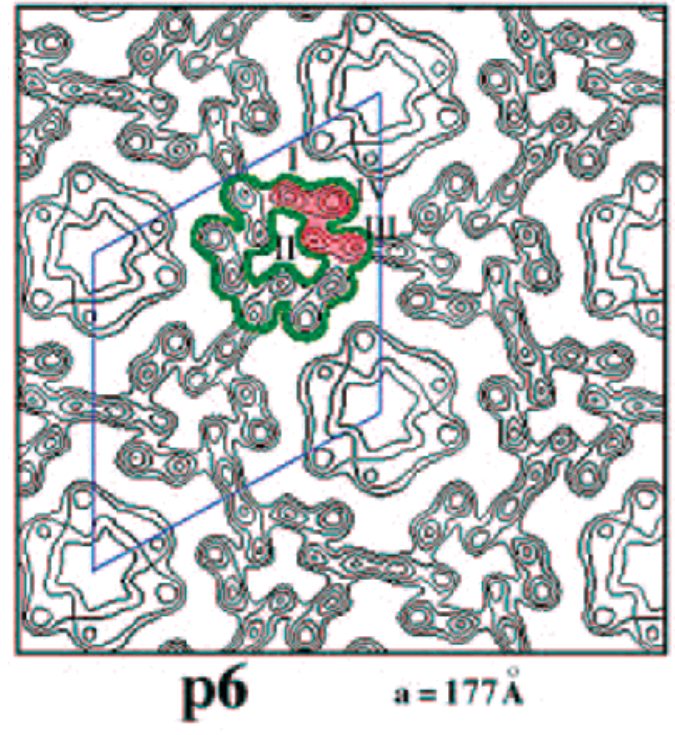

(a)

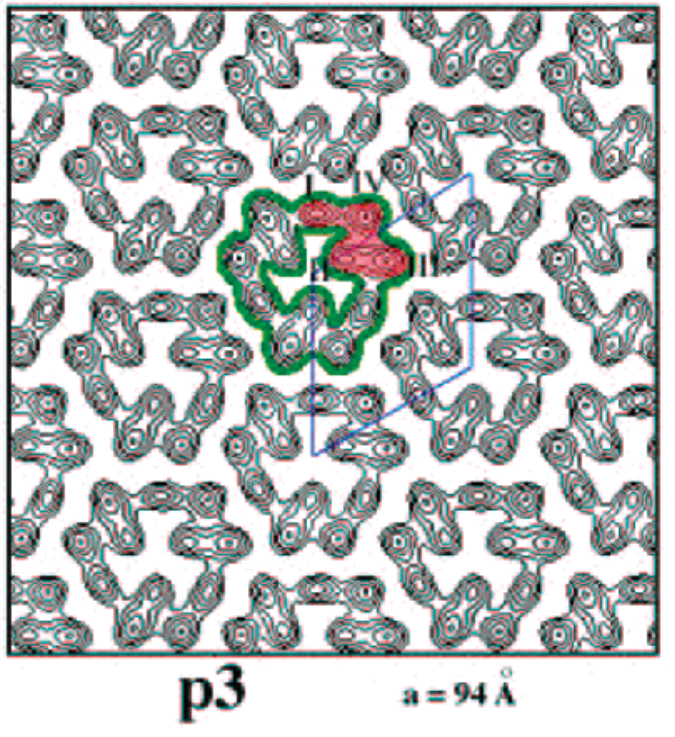

(b)

Figure 1. Two-dimensional projection maps of the structure of (a) the p6 and (b) the p3 phases of annexin $\mathrm{V}$, at $20 \AA$ resolution. In each plot, one trimer is outlined and one annexin $\mathrm{V}$ molecule is shaded. The four domains are numbered according to Huber et al. (ref 22). The figure also indicates the lattice cell dimension a. Note that in the p6 phase, the presence of the central trimer enclosed within the 6-fold symmetry cage depends on the surface coverage.

a rigid hexagonal lattice of trimers, in which each cell can contain a rather mobilecentral trimer (seeFigure1). This phase is named 06 due to its apparent p6 symmetry although, in fact, the symmetry is lower since not every cell contains a "captured" trimer and, if it is present, the mobile central trimer does not exhibit 6-fold symmetry. At a higher density of binding sites, another crystalline form becomes more stable, where the trimers are packed moretightly, sothat each particleis surrounded by nearest neighbors arranged in a p3 symmetry class. ${ }^{9}$ Thetransition to thehigher density structure is induced experimentally by stressing the system, for example, by transferring the films from an air-water interface to an EM grid (equivalent to increasing the lateral pressure) or by increasing theconcentration of theli pid ligand in solution (equivalent to increasing the surface density of trimers). There is experimental evidence which suggests that the p6-p3 transition is first order, and its reversibility has been monitored using AFM; the transition is characterized by an increase in surface density of trimers of about $15 \% .^{12}$

Two-dimensional aggregation of membraneproteins has been approached with simple models since the 1990s. Sperotto and Mouritsen have applied Monte Carlo simulations and mean field calculations to a statistical me chanical lattice model of lipid-protein interactions in membranes in order toinvestigatethephase equilibria. ${ }^{13,14}$ A good summary of protein aggregation in membrane systems has been given by Bruinsma and Pincus. ${ }^{15}$ They focused on aggregation in membranes induced by membrane-mediated elastic deformation and concluded that

(12) Reviakine I : Bergsma-Schutter, W. Morozov, A. N.; Brisson A. Two-dimensional crystallization of annexin A5 on phospholipid bilayers and monolayers: a solid-solid phasetransition between crystal forms. Langmuir 2001, 17, 1680-1686.

(13) Sperotto, M. M.; Mouritsen, O. G. Mean field and Monte Carlo simulation studies of thelateral distribution of proteins in membranes. Eur. Biophys. J . 1991, 19, 157-168.

(14) Sperotto, M. M. A theoretical model for the association of amphiphilic transmembrane peptides in lipid bilayers. Eur. Biophys. J. 1997, 26, 405-416.

(15) Bruinsma, R.; Pincus, P. Protein aggregation in membranes. Curr. Opin. Solid State Mater. Sci. 1996, 1, 401-406. aggregation may occur if the protein inclusion suffers a thickness mismatch, induces a local changein membrane stiffness, or acts as gap junction pinching together the membranes. The effect of lipid-protein interactions in the protein aggregation has been reviewed morein detail by $\mathrm{Gil}$ and co-workers. ${ }^{16}$ On the basis of a variety of theoretical considerations and model calculations, the nature of lipid-protein interactions is considered both for a single protein and for an assembly of proteins that can lead to aggregation and protei $n$ crystallization in the plane of the membrane. The phenomena include lipid sorting and selectivity at protein surfaces, protein-lipid phase equilibria, lipid-mediated protein-protein interactions, wetting and capillary condensation as means of protein organization, mechanisms of two-dimensional protein crystallization, and nonequilibrium organization of active proteins in membranes. More recently, Dommersnes and Fournier ${ }^{17}$ have studied the collective behavior of membrane inclusions in a flexible fluid membrane. In thesecalculations, thelong-rangeattractive interactions between inclusions arefound to besufficiently strong to induce aggregation. However, as we shall see, the main result of our study is that the long-range interactions do not need to be included to reproduce the p3 and p6 phases for annexin $\mathrm{V}$.

Beforewe describethemodeand simulation of annexin $\mathrm{V}$, wenotethat thereareother examples of proteins giving periodicphases in two dimensions, for example, the purple phase of aggregated bacteriorhodopsin, 18 where the geometry consists of hexagonal close packing. Although the model we describe is valid for annexin $\mathrm{V}$, the general treatment is valid for any two-dimensional system with

(16) Gil, T.; I psen, J . H.; Mouritsen, O. G.; Sabra, M. C.; Sperotto M. M.; Zuckermann, M.J . Theoretical analysis of protein organization in lipid membranes. Biochim. Biophys. Acta Rev. Biomembranes 1998 $1376,245-266$.

(17) Dommersnes, P. G.; F ournier, J . B. N-body study of anisotropic membrane inclusions: Membrane mediated interactions and ordered aggregation. Eur. Phys. J . B 1999, 12, 9-12.

(18) Baudry, J .; Tajkhorshid, E.; Molnar, F.; Phillips, J .; Schulten, $\mathrm{K}$. Molecular dynamics study of bacteriorhodopsin and the purple membrane. J . Phys. Chem. B. 2001, 105, 905-918. 


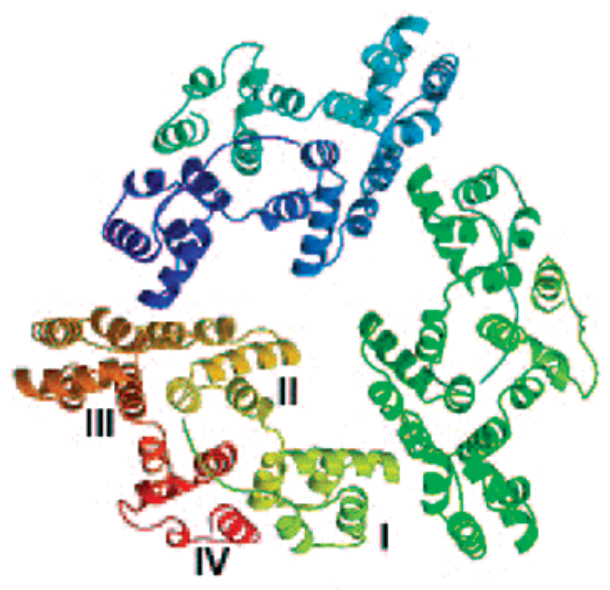

(a)

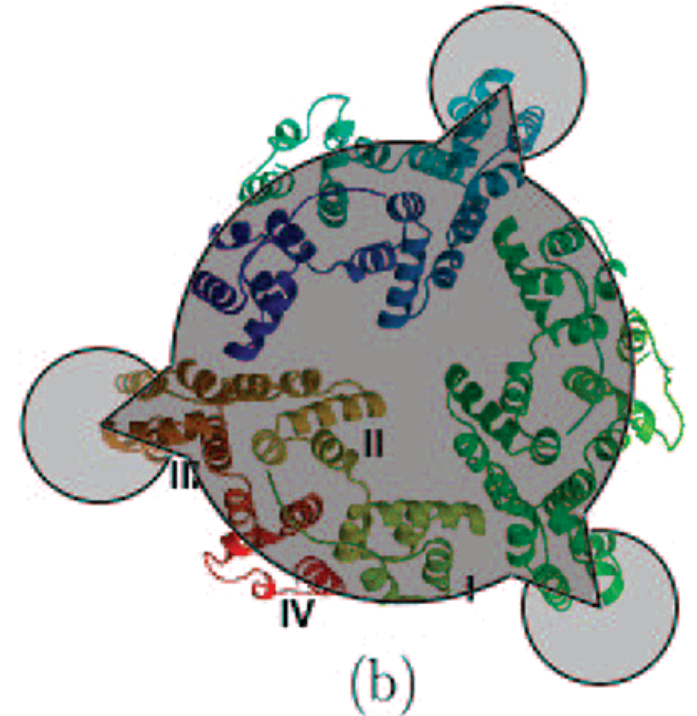

(b)

Figure 2. (a) Molecular structure of annexin $V$ trimer (ref 23) and (b) the simplified model, with the approximate location of the domains of the real trimer numbered according to Huber et al. (ref 22). The light gray regions indicate the location and range of the attractive interactions which occur between the domains III in pairs of the real protein, and the darker gray central region indicates the excluded area "hard body" of the model potential.

specific, local ized interactions, that is, when the relevant protein-protein interactions can be described by (highly directional) short-rangeforces. Therearecases wherelongrange forces are important. This could be the case for other protein inclusions which strongly modify the elasticity of the supporting membrane.

\section{Simplified Model for the 2D Crystallization of Annexin V}

Thetrimer structure of thesurface-bound proteins (see Figure 2) and the organization of these within the $p 6$ and p3 phases (see Figure 1) suggests the essential features of a simplified model potential. The electron microscopy map of the 6 phase shows clearly that thetrimer-trimer interactions are extremely localized between adjacent domains III of neighboring molecules and that these domains must beal most perfectly al igned. I n other words, the interactions should be extremely localized, short ranged, and orientation dependent. Consideration of the p3 phase, on the other hand, suggests that at higher density the trimers need to rearrange in order to accommodate particles in a more efficient space-filling way, rotating such that the domains III of a triplet of trimers interact at $120^{\circ}$ as opposed to $180^{\circ}$ for a pair of trimers in the 06 phase.

These observations lead us to formulate a simplified version of the trimers which is pictured in Figure 2 . The internal structure of thetrimer is taken to befrozen; thus, the shape of the trimer is taken to be a rigid hard body model composed of an equilateral triangleand a hard disk sharing the same center, with the diameter of the circle the same as the height of the triangle $\sigma=\mathrm{h}$. The three vertexes of thetriangle represent thelocation of thethree domains III in the real trimer. Short-range attractions $r$ $=\mathrm{h} / 6$ are located at the vertexes, which are modeled as a simple linear ramp increasing from $-\epsilon$ to zero. As discussed earlier, directionality of the attractive interactions is important, as it singles out two preferred orientations at $120^{\circ}$ ( $p 3$ phase) and $180^{\circ}$ (p6 phase). The actual functional form of the angle dependence is not important for our model so long as these two angles are preferred, but the EM maps and molecular mechanics calculations suggest that three main ingredients should be included: preferred interaction angles between the domains III at $120^{\circ}$ and $180^{\circ}$, comparable interaction energies when two trimers interact at $180^{\circ}$ (p6 phase) and threeinteract at $120^{\circ}$ (p3 phase), and a relativeenergy penalty for any other angles, especially less than $90^{\circ}$. We choose the angular-dependent potential to be

$$
\mathrm{u}_{2}(\theta)= \begin{cases}1 & \theta<90^{\circ} \\ \cos \theta+\frac{1}{2}(1-\cos 6 \theta) & 90^{\circ}<\theta<270^{\circ} \\ 1 & \theta>270^{\circ}\end{cases}
$$

Full details of the potential and the simulations, as well as a theoretical study of the p6 phase, will be given elsewhere. ${ }^{19}$ The important point here is that as the potential is somewhat generic, any potential of similar form should reproduce the experimental phase behavior, so long as the correct physics enters the potential model.

\section{Computer Simulation of the Model Trimers}

Systems of model timers were studied using grand canonical (constant chemical potential or osmoticpressure) Monte Carlo simulations. ${ }^{20}$ Rather than using a fixed number of molecules, which is common for many simulations, thegrand canonical ensembleuses a varying amount of molecules, but with the average amount governed by the chemical potential. This simulation technique is particularly attractive for this model, since it mimics the behavior at the surface in the real experiments during the formation of the p6 phase. Thus, by altering the chemical potential of surface trimers in the simulation, which is equivalent to controlling the concentration or osmotic pressure of the protein in solution in the real experiments, the surface coverage can be varied. The advantage of the grand canonical simulations over other common simulation techniques is that we can simulate an "open" system, in which the number of particles is not fixed. Our system is open in the sense that we are

(19) Bates, M. A.; Noro, M. G.; Frenkel, D. Computer simulation of the phasebehaviour of a model membraneprotein: annexin V.J . Chem. Phys., submitted.

(20) Smit, B.; Frenkel, D. Understanding Molecular Simulations; Academic Press: San Diego, 1996. 


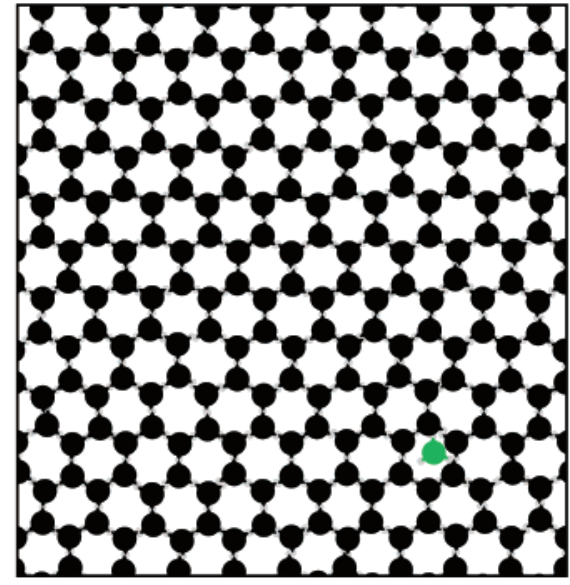

(a)

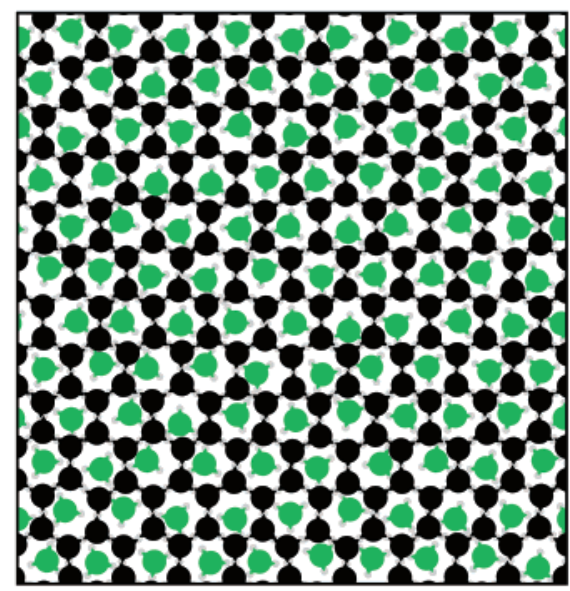

(c)

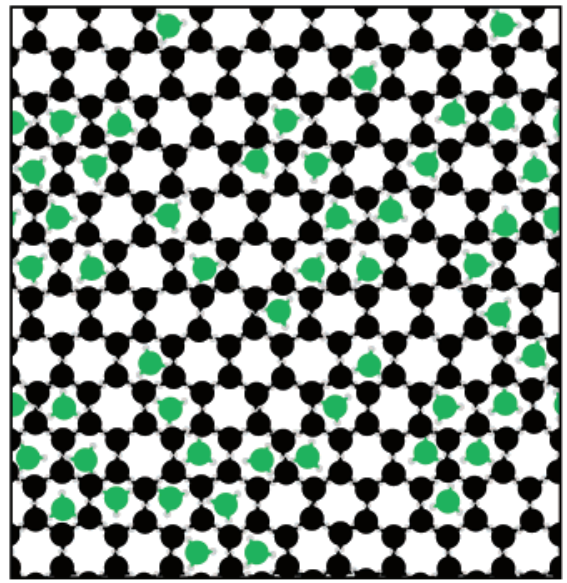

(b)

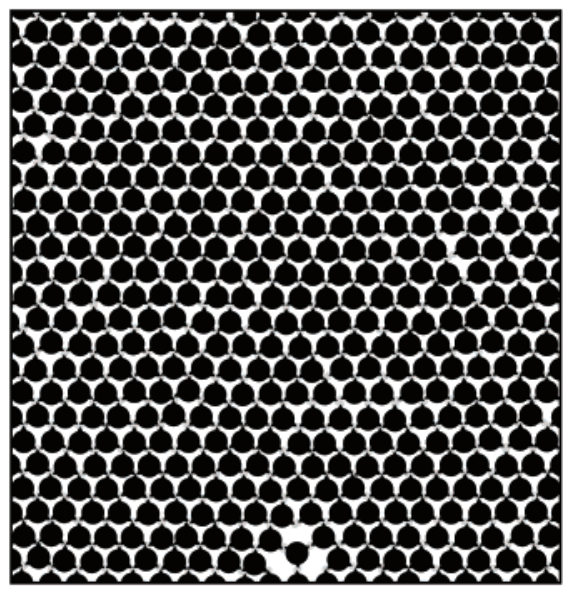

(d)

Figure 3. Configurations from the simulations of model annexin $\mathrm{V}$. The p6 phase at (a) low, (b) intermediate, and (c) high surface coverageand (d) thep3 phase. For clarity, the captured central trimers in the p6 phasehavebeen col ored differently tothoseforming the hexagonal lattice. Note the "p6-like" defect in the p3 phase. At low temperature, these are rel atively uncommon; however, at high temperaturethey are more common and can frequently be observed al ong linedefects between different regions of p3 structure.

simulating only the surface-adsorbed proteins and not the proteins in solution; this means that adsorption (or desorption) of a protein trimer onto (from) the surfacecan bemodeled by theaddition (or removal) of a model trimer from the simulation.

At low protein concentration, the surface coverage is low, and the adsorbed proteins hardly interact. If the chemical potential is increased past a certain threshold, a large jump in the surface density is observed. The resulting structure is shown in Figure 3a; the system clearly forms an open hexagonal structureof p6 symmetry. As thechemical potential is increased, thedensity is found to increase conti nuously up to a critical value. Similarly, if the chemical potential is decreased, the coverage decreases continu ously down tothat of theopen hexagonal lattice. Exampleconfigurations at intermediateand higher coverage are shown in Figure 3b,c and are clearly comparable to that shown in Figure 1. We observe that thesurfacecoverageincreases si mply by adsorbing trimers into the unoccupied cavities in the hexagonal lattice. Similarly, if the chemical potential is lowered, it is the central trimers which are desorbed, and the hexagonal lattice remains intact.

However, once the surface coverage reaches a certain limit, with all the hexagonal cavities filled, thereappears to be noway of absorbing further trimers ontothe surface. However, thesimulations indicatethat there is a coverage jump of $15 \%$ as thechemical potential is further increased. Figure $3 d$ shows how this can occur. The hexagonal structure observed previously is destroyed, in favor of a structure with $\mathrm{p} 3$ symmetry, reminiscent of the $\mathrm{p} 3$ phase of annexin $\mathrm{V}$ (see Figure 1). This phase is more efficient at packing than the p6 structureand so is stableat higher densities. Note that, in our model, we choose the energy of a p6-type bond $\left(180^{\circ}\right)$ to be twice that of a p3-type bond $\left(120^{\circ}\right)$; since each trimer interacts with three others in the (empty) p6 phase and six others in the p3 phase, the potential energy of these phases will be equal. However, since $1 / 7$ of the trimers in the full p6 phase are noninteracting (the captured ones), the p3 phase is al so slightly energetically favored at high density in comparison to the "filled" p6 phase. Thus, this transition is most probably partly energy and partly entropy (or packing) driven.

The simulations described are performed at a single finite temperature, $\mathrm{kT} \sim \epsilon$, and the system is found to reproduce the experimentally observed phase behavior. In a simulation, we need not be constrained by the temperatures usually accessi blefor an experiment and so can study extreme limits for the model, which would not be accessible in the laboratory. However, limits such as 
very high temperatures areusually not so interesting since thesewould correspond to conditions at which, for example, the individual trimers would behave differently and not form trimers or possibly even decompose. Therefore, here weonly describesimulations at reasonabletemperatures. However, we have computed the phase diagram as a function of temperature and this will be presented el sewhere, along with a simple theory for the behavior of the 6 phase. ${ }^{19}$ We should al so point out that whilewe use a simple potential defined by a given set of parameters, variation in the parameters should lead to very similiar phase diagrams so long as the essential physics of the interactions described in section II are upheld; that is, the potential must possess the correct 3-fold symmetry, theangul ar potential should favor thecorrect al ignments, and the attractive interactions are short range. Indeed, if the range of the attractive interactions is chosen to be of the order of the trimer size, the phase behavior is dramatically modified, leading to a 2D liquid-vapor type transition, as we expect. ${ }^{21}$

(21) Noro, M. G.; Frenkel, D. Phase behavior of a simple model for membrane proteins. J . Chem. Phys. 2001, 114, 2477-2483.

(22) Huber, R.; Römisch, J. M.; Paques, E. P. The crystal and molecular structure of human annexin $\mathrm{V}$, an anticoagulant protein that binds to calcium and membranes. EMBO J . 1990, 9, 3867-3874.

\section{Conclusions}

The highly simplified model for annexin $V$ described here, based only on hard core interactions plus highly directional short-range interactions, is able to reproduce the qualitative phase behavior of such a complex membrane binding protein in a surprisingly good agreement with experiments. It is also able to reproduce quantitatively the change in surface coverage of $15 \%$ at the first transition between the $p 6$ and $p 3$ phases. These results indicatehow relatively crude, generic models which strip away the complicated chemical detail but retain the essential physics of the interactions between large molecules can be used to understand the formation of phase structures such as the p6 and p3 phases of annexin V.

Acknowledgment. The work of the FOM Institute is part of the research program of "Stichting Fundamenteel Onderzoek der Materie" (FOM) and is supported by NWO. This work was made possible through EU Grant ERBFMBICT982949 and through funding of thePPM-CMS Project 96PPM 003.

\section{LA0156356}

(23) Sopkova, J .; Renouard, M.; Lewit-Bentley, A. The crystal structure of a new high calcium form of annexin V. J. Mol. Biol. 1993, $234,816-825$. 